\title{
Primary cutaneous precursor B-cell lymphoblastic lymphoma with concomitant skin and nodal involvement in a child
}

\author{
Houda Hammami ${ }^{1}$, Amal Chamli ${ }^{1}$, Raja Jouini ${ }^{1}$, Rym Benmously ${ }^{1}$, Ashraf Debbiche ${ }^{1}$, \\ Anissa Zaouak ${ }^{1}$, and Samy Fenniche ${ }^{1}$ \\ ${ }^{1}$ University of Tunis El Manar Faculty of Medicine of Tunis
}

September 24, 2021

\begin{abstract}
Primary cutaneous B-cell lymphoblastic lymphoma (PB-LBL) is an exceedingly rare malignant neoplasm in children. Skin lesions may represent the initial sign. Herein, we report a case of a PB-LBL with cutaneous lesions as a primary extranodal involvement, occurring in a 5-year-old infant.
\end{abstract}

Title : Primary cutaneous precursor B-cell lymphoblastic lymphoma with concomitant skin and nodal involvement in a child

Running title: B-cell lymphoblastic lymphoma

Authors Houda Hammami ${ }^{1,2}$, MD, Amal Chamli ${ }^{1,2}$, MD, Raja Jouini ${ }^{2,3}$, MD, Rym Benmously ${ }^{1,2}$, MD, Ashraf Debbiche ${ }^{2,3}, \mathrm{PhD}$, Anissa Zaouak ${ }^{1,2}, \mathrm{MD}$, Samy Fenniche ${ }^{1,2}, \mathrm{PhD}$

Affiliations:

1-Dermatology Department, Habib Thameur Hospital, Tunis, Tunisia

2-University of Tunis El Manar, Faculty of medicine of Tunis, Tunisia

3-Pathology Department, Habib Thameur Hospital, Tunis, Tunisia

Corresponding author: Amal Chamli, MD

Address: Habib Thameur Hospital, 8 Ali Ben Ayed,

Montfleury, 1008, Tunis, Tunisia

E-mail:amal.fmt@gmail.com

ORCID : https://orcid.org/0000-0002-7927-9088

Tel: 0021654085688

Funding: none

Conflict of interest: none

Acknowledgements: none

Authorship: All authors had access to the data and a role in writing this manuscript.

Table count: 0 
Figures: 1

Word count: 506

References: 4

Key words: Dermatology, Hematology, Paediatrics and adolescents medicine

Primary cutaneous precursor B-cell lymphoblastic lymphoma with concomitant skin and nodal involvement in a child

\begin{abstract}
:
Primary cutaneous B-cell lymphoblastic lymphoma (PB-LBL) is an exceedingly rare malignant neoplasm in children. Skin lesions may represent the initial sign. Herein, we report a case of a PB-LBL with cutaneous lesions as a primary extranodal involvement, occurring in a 5-year-old infant.
\end{abstract}

Key words: lymphoma, non-Hodgkin lymphoma, lymphoblastic, B-cell lymphoma, pediatric

\title{
Key Clinical Messages:
}

- Skin lesions may represent the initial sign of primary cutaneous B-cell lymphoblastic lymphoma (PB-LBL).

- PB-LBL should be considered as a potential differential diagnosis for head and neck tumors especially in the pediatric population.

\section{Case report}

A 5-year-old previously healthy boy presented to our dermatology department with a 2-month history of skin tumor on his scalp associated with cervical nodes. On physical examination, there was a $4 \mathrm{~cm}$, dome-shaped, firm, tender, erythematous tumor of the left parietal region of the scalp (figure 1). It was associated with multiple, bilateral, asymmetric cervical nodes. Blood cell count showed normal values for blood cell count and platelet count. Skin biopsy of the scalp lesion showed a diffuse infiltrate of atypical lymphoid cells in the entire dermis (figure 2A). The neoplastic lymphoid cells appeared medium-sized with inconspicuous nucleoli, scant cytoplasm, and a high mitotic rate (figure 2B). A grenz zone was present below a normalappearing epidermis. Immunohistochemical (IHC) studies revealed that neoplastic cells expressed terminal deoxynucleotidyl transferase (TdT) (figure 2C), CD10, CD34, CD79a, and Bcl2, but were negative for CD3, and CD20. Peripheral blood and bone marrow aspiration smear, cerebrospinal fluid analysis revealed no evidence of any neoplastic populations. Whole-body computed tomography revealed involvement of the cervical, axillary and mesenteric lymph nodes associated with hepatomegaly. Based on these findings a diagnosis of primary cutaneous precursor B-cell lymphoblastic lymphoma was made. The patient was treated with polychemotherapy GFAL B2001 protocol including 6 courses of vincristine, oncovin, methotrexate, and prednisone. He showed partial remission after a 14-month follow-up.

\section{Discussion:}

Lymphoblastic lymphoma (LBL) is an uncommon non-Hodgkin lymphoma and accounts for only $5 \%$ of all non-Hodgkin's lymphoma. According to the WHO classification, LBL is distinguished from acute lymphoblastic leukemia (ALL) by a bone marrow blast count of less than $20 \%$ of nucleated cells ${ }^{1}$. It can be of T-cell or B-cell lineage. B-cell LBL (B-LBL), the rarer subtype, is a high-grade neoplasm of immature B cells that demonstrate lymphoblastic morphology and express precursors and B-cell markers ${ }^{2}$. It accounts for less than $10 \%$ of cases of lymphoblastic lymphoma. In contrast to T-cell LBL, B-LBL tends to involve skin and bone and to spare mediastinal lymph nodes. Primary cutaneous precursor B-LBL (PB-LBL) is exceedingly uncommon in children and seems to occur more frequently in young females ${ }^{3}$. It usually presents as solitary red to purple nodules with a propensity for the head and neck area ${ }^{4}$.

Histologically, there is an overlap between B-LBL and other lymphoid malignant neoplasms mainly precursor T-cell LBL, and 'small blue round cell' neoplasms such as Ewing's sarcoma, rhabdomyosarcoma, and neuroblastoma, rendering histopathological diagnosis difficult. Thus, IHC is crucial and very useful to rule 
out differential diagnoses. Indeed, the expression of TdT and CD34 and the absence of CD20 markers on B-lineage lymphomas suggest their precursor B-cell origin, as in our case ${ }^{3}$.

Although PB-LBL is a highly aggressive malignant neoplasm, its prognosis is dramatically improved with the use of aggressive multi-agent chemotherapy ALL-regimens with up to $90 \%$ of 5-year EFS rate ${ }^{3}$. Partial remission, as seen in our case despite an aggressive multi-agent systemic chemotherapy, is rare.

In conclusion, this case serves as an important reminder for clinicians to consider this entity as a potential differential diagnosis for head and neck tumors especially in the pediatric population.

\section{Conflicts of Interest: None declared}

\section{Funding sources: None}

Ethics statement: Appropriate consent has been obtained, prior to submission, for the publication of images and data.

Data availability statement: Data sharing was not applicable to this article as no datasets were generated or analyzed during the current study.

\section{Author Contributions:}

Drs Houda Hammami and Amal Chamli contributed to the first draft of the manuscript. Drs Houda Hammami, Amal Chamli, Raja Jouini, and Rym Benmously, contributed to the literature search, analysis, and interpretation of the data. Drs Ashraf Debbiche, Anissa Zaouak, and Samy Fenniche critically revised the manuscript and gave final approval. All authors read and approved the final manuscript and agree to be finally accountable for ensuring the work's integrity and accuracy.

\section{Acknowledgements: None}

\section{References}

1. Swerdlow SH, Campo E, Pileri SA, et al. The 2016 revision of the World Health Organization classification of lymphoid neoplasms.Blood. 2016;127(20):2375-2390.

2. Kawakami T, Kimura S, Kinoshita A, Kondo K, Soma Y. Precursor B-cell Lymphoblastic Lymphoma with only Cutaneous Involvement. Acta Derm Venerol . 2009;89(5):540-541.

3. Ceppi F, Pope E, Ngan B, Abla O. Primary Cutaneous Lymphomas in Children and Adolescents: Cutaneous Lymphomas in Children and Adolescents. Pediatr Blood Cancer . 2016;63(11):1886-1894.

4. Lee WJ, Moon HR, Won CH, et al. Precursor B- or T-lymphoblastic lymphoma presenting with cutaneous involvement: A series of 13 cases including 7 cases of cutaneous T-lymphoblastic lymphoma. J Am Acad Dermatol . 2014;70(2):318-325.

Figure legend:

Figure 1: a $4 \mathrm{~cm}$, dome-shaped erythematous tumor of the left parietal region of the scalp in a 5-year-old child.

Figure 2: $\mathbf{A}$ and B, skin biopsy showed a diffuse infiltrate in the entire dermis, composed of medium-sized lymphoid cells with inconspicuous nucleoli, scant cytoplasm, and a high mitotic rate (Haematoxylin and Eosin $\times 200$ and $\times 600$, respectively), C, Immunohistochemical staining revealed positive terminal deoxynucleotidyl transferase cells $(\mathrm{TdT}, \times 200)$. 

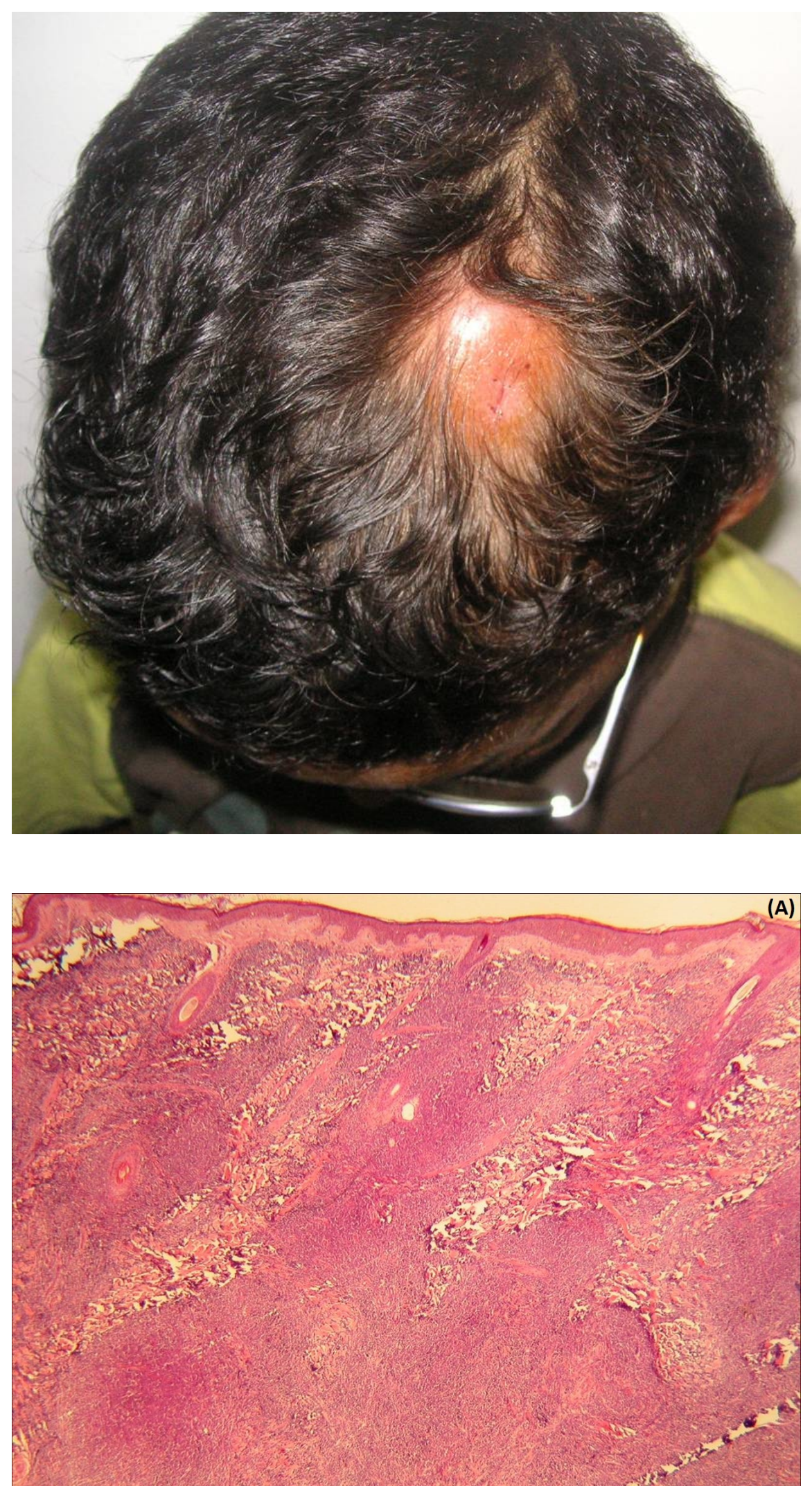


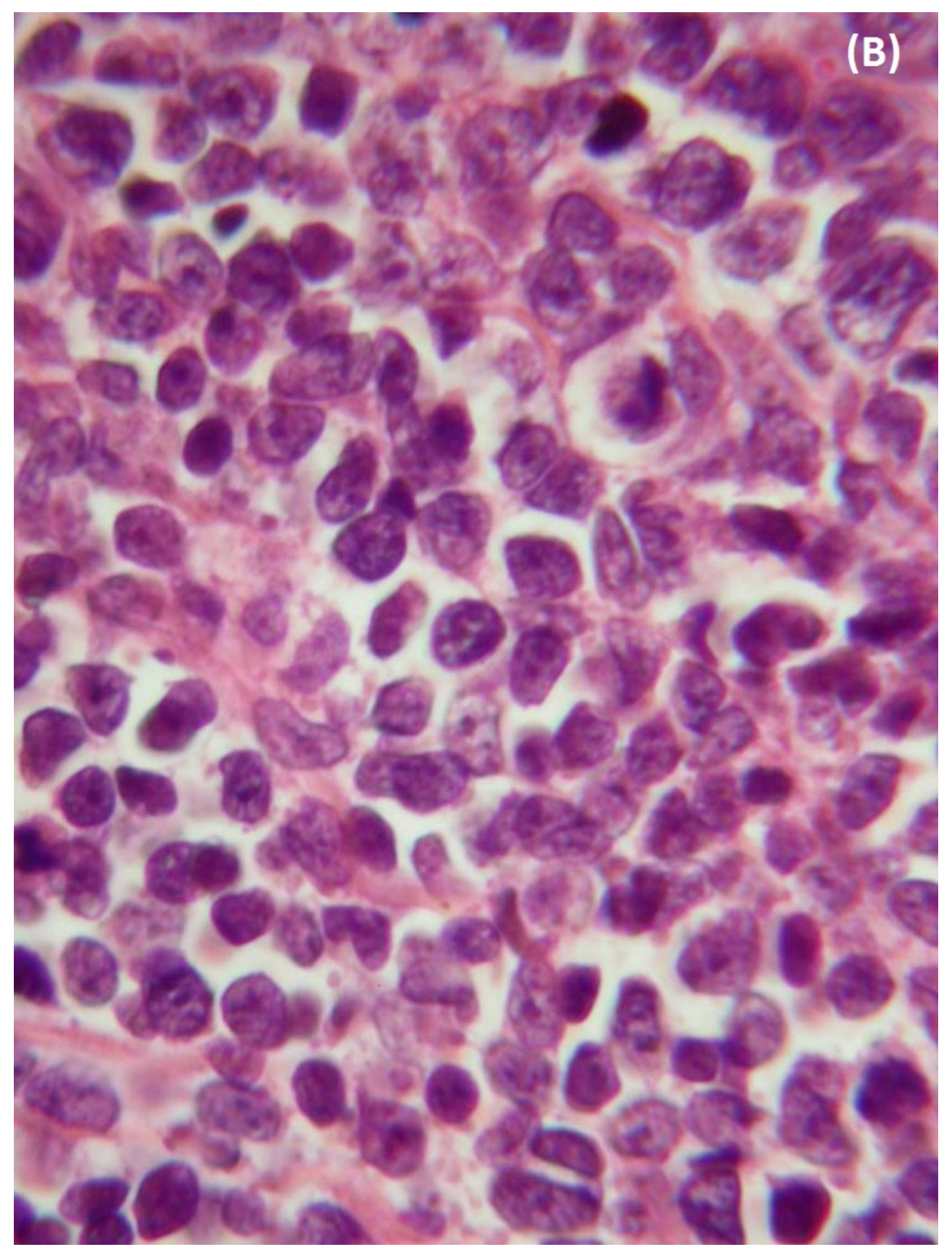




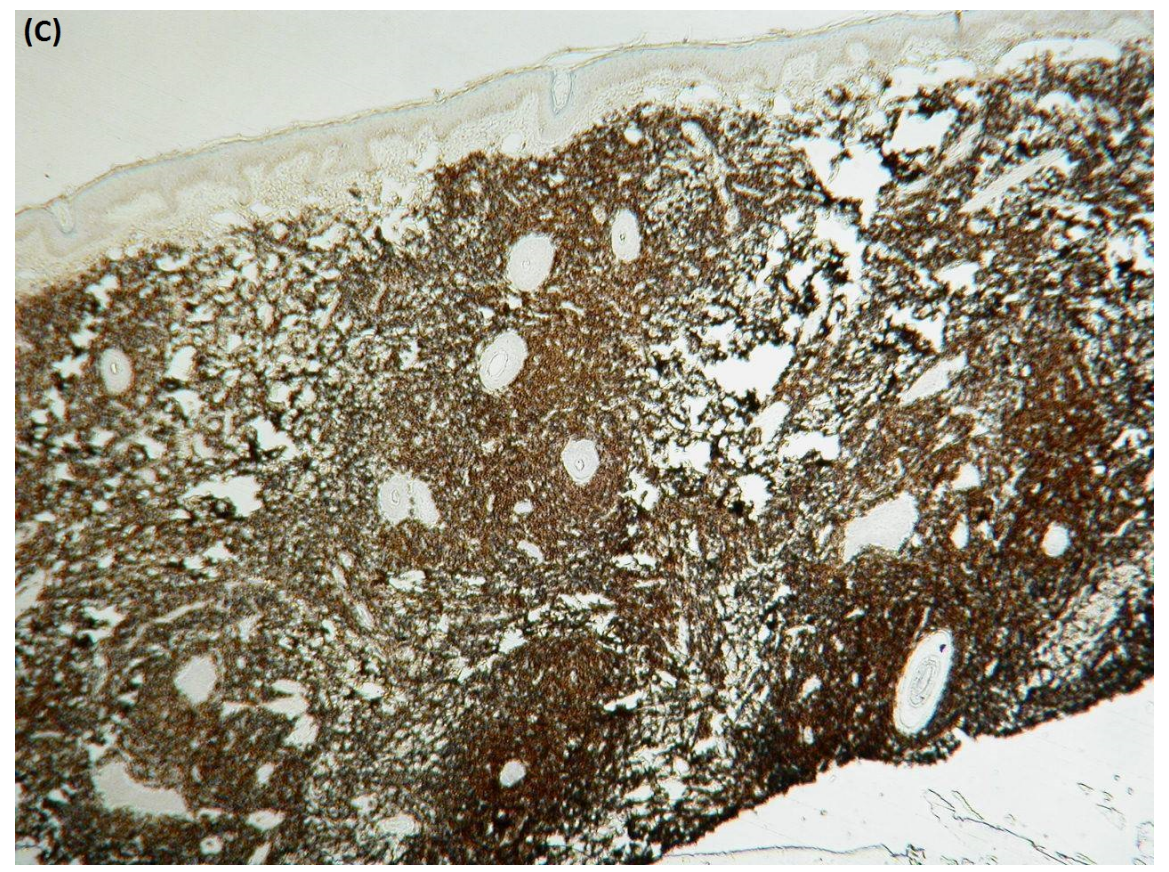

